\title{
Sub-Arcsecond Morphology of Planetary Nebulae
}

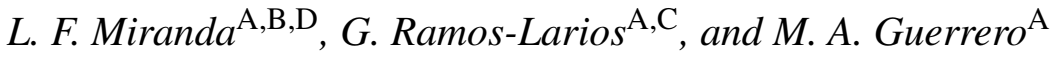 \\ A Instituto de Astrofísica de Andalucía - CSIC, Granada, Spain \\ ${ }^{\text {B }}$ Facultade de Ciencias, Universidade de Vigo, Vigo, Spain \\ ${ }^{\mathrm{C}}$ Instituto de Astronomía y Meteorología, Guadalajara (Mexico) \\ D Corresponding author. Email: 1fm@iaa.es
}

Received 2009 June 28, accepted 2010 February 11

\begin{abstract}
Planetary nebulae (PNe) can be roughly categorized into several broad morphological classes. The high quality images of PNe acquired in recent years, however, have revealed a wealth of fine structures that preclude simplistic models for their formation. Here we present narrow-band, sub-arcsecond images of a sample of relatively large PNe that illustrate the complexity and variety of small-scale structures. This is especially true for bipolar PNe, for which the images reveal multi-polar ejections and, in some cases, suggest turbulent gas motions. Our images also reveal the presence or signs of jet-like outflows in several objects in which this kind of component has not been previously reported.
\end{abstract}

Keywords: circumstellar matter — ISM: jets and outflows — planetary nebulae

\section{Introduction}

Planetary nebulae (PNe) consist of material ejected from the Asymptotic Giant Branch (AGB) progenitor, which is photoionized by the hot central star and swept up by the fast stellar wind (Kwok, Purton \& Fitzgerald 1978). The morphology of PNe has attracted the attention of many observers because it is related to and contains information about the processes of mass ejection involved in their formation. A number of investigations have been devoted to the morphological classification of PNe (Balick 1987; Schwarz, Corradi \& Melnick 1992; Stanghellini, Corradi \& Schwarz 1993; Manchado et al. 1996). In general, three broad morphological classes have been considered: round (or circular), elliptical and bipolar. The formation of the three classes was explained within the Generalized Interacting Stellar Winds (GISW) Model (Balick 1987) assuming an azimuthal dependence of the density in the slow wind, having its maximum at the equatorial plane. Bipolar and elliptical $\mathrm{PNe}$ would present 'high' and 'intermediate' density contrasts, while round PNe would present a homogeneous slow wind. Numerical simulations have shown that the three main morphological classes can be reproduced under this hypothesis (e.g. Frank et al. 1993).

The detection of collimated outflows in PNe (e.g. Gieseking, Becker \& Solf 1985; Miranda \& Solf 1992) poses a serious difficulty to the GISW model because this model is unable to explain the presence of highly collimated ejections. Moreover, no collimating agent was foreseen in these evolutionary phases, which could cause high collimation. In addition, the collimated ejections in most PNe present different orientations with respect to the central star suggesting that the collimated agent can precess or wobble (Miranda \& Solf 1992; Miranda, Guerrero \& Torrelles 1999; Guerrero et al. 2008).

More recently, high-resolution images obtained with the Hubble Space Telescope HST have shown that PNe are highly complex objects and that simplistic models are unable to account for the large variety of structures and microstructures observed in these objects. In particular, the signature of collimated outflows appears in a very large fraction of PNe (e.g. Sahai \& Trauger 1998). Many PNe show multiple bipolar ejections with different geometries that require episodic ejections (e.g. Guerrero, Chu \& Miranda 2004). These observations have led to suggest that collimated outflows are the basic mechanism that shapes PNe (Sahai \& Trauger 1998). Nevertheless, other physical phenomena should be also present in PN formation as wind interaction, magnetic fields and/or the binary nature of the central star (see Balick \& Frank 2002). Although a large number of PNe have already been imaged (Balick 1987; Schwarz et al. 1992; Manchado et al. 1996; Sahai \& Trauger 1998), in some cases the images lack spatial resolution and/or quality to enable a proper identification of the morphological components. In fact, images at higher resolution and/or deeper than previous ones continue discovering new structures, even in well observed $\mathrm{PNe}$, which result to be key for a proper interpretation of their formation (e.g. Maestro, Guerrero \& Miranda 2004; Miranda et al. 2006). These results and the complexity of $\mathrm{PNe}$ clearly justify the imaging of $\mathrm{PNe}$ whose images are of insufficient quality.

In this paper, we present narrow-band images of a sample of relatively large PNe, including elliptical and bipolar PNe. The images have been taken under particularly good seeing conditions and reveal novel details in the objects. 


\section{Sample Selection and Observations}

The observed PNe were selected from the Manchado et al. (1996) catalog among those with no independent $\mathrm{H} \alpha$ and [NII] images and/or with low resolution images due to poor seeing conditions. Images isolating the $\mathrm{H} \alpha$ and [NII] lines are crucial because these two emission lines may trace very different regions of the nebulae; in particular, collimated outflows and microstructures can be better recognized and studied in [NII]. In addition, good seeing conditions allow us a detailed morphological study of the structures present in a PN. We have added IC 5217 and NGC 6778 to this sample in order to study at high spatial resolution the structures identified by Miranda et al. (2006) and Maestro et al. (2004), respectively.

Direct images were obtained on 2006 June and 2008 September using ALFOSC at the $2.56 \mathrm{~m}$ Nordic Optical Telescope on Roque de los Muchachos Observatory (La Palma, Spain). A $2 \mathrm{~K} \times 2 \mathrm{~K}$ E2V CCD with plate scale of $0.19^{\prime \prime}$ pixel $^{-1}$ was used as detector. We used three narrow-band filters that isolate the light of $\mathrm{H} \alpha(\Delta \lambda=9 \AA)$, [NII] $\lambda 6584$ (hereafter $[\mathrm{NII}])(\Delta \lambda=9 \AA)$ and $[\mathrm{OIII}] \lambda 5007$ (hereafter [OIII]) $(\Delta \lambda=30 \AA)$. Exposure time per filter was $900 \mathrm{~s}$ or $1800 \mathrm{~s}$. The seeing during the observations was between $0.5^{\prime \prime}$ and $0.9^{\prime \prime}$. The images were reduced using standard IRAF routines.

\section{Results and Discussion}

In the following we will discuss the images of the objects observed. In some cases, we have grouped together some objects because of their morphological similarities.

\subsection{IC 351 and Vy 1-1}

In the Manchado et al. (1996) catalog, IC 351 is classified as an elliptical PNe with internal structures while Vy 1-1 is classified as an elliptical multiple-shell PN. The two objects are not included in the lists of PNe with low-ionization structures, knots or jets by Goncalves, Corradi \& Mampaso (2001).

Figures 1 and 2 show our images of IC 351 and Vy 1-1, respectively. The two $\mathrm{PNe}$ present noticeable morphological similarities, including low-ionization polar structures which are identified in these images for the first time.

IC 351 is a high-excitation PN consisting of an inner elliptical shell oriented along PA $\sim 355^{\circ}$ surrounded by an outer round attached shell. The central star is detected in the [NII] and [OIII] filters. The [NII] image shows two collimated structures emanating from bright polar caps of the inner elliptical shell that extend $\sim 3^{\prime \prime}$ and end in bright knots. The $\mathrm{H} \alpha$ and [OIII] images also show hints of these structures but they are relatively much fainter, implying low-excitation.

Vy 1-1 (Figure 2) shows an inner elliptical shell oriented at $\mathrm{PA} \sim 70^{\circ}$, and an outer round attached shell. Although no [OIII] image has been obtained for Vy 1-1, the faint emission in [NII] and the bright emission in [OIII] (see Manchado et al. 1996) indicate that both shells are of high-excitation. The polar regions of the inner shell are
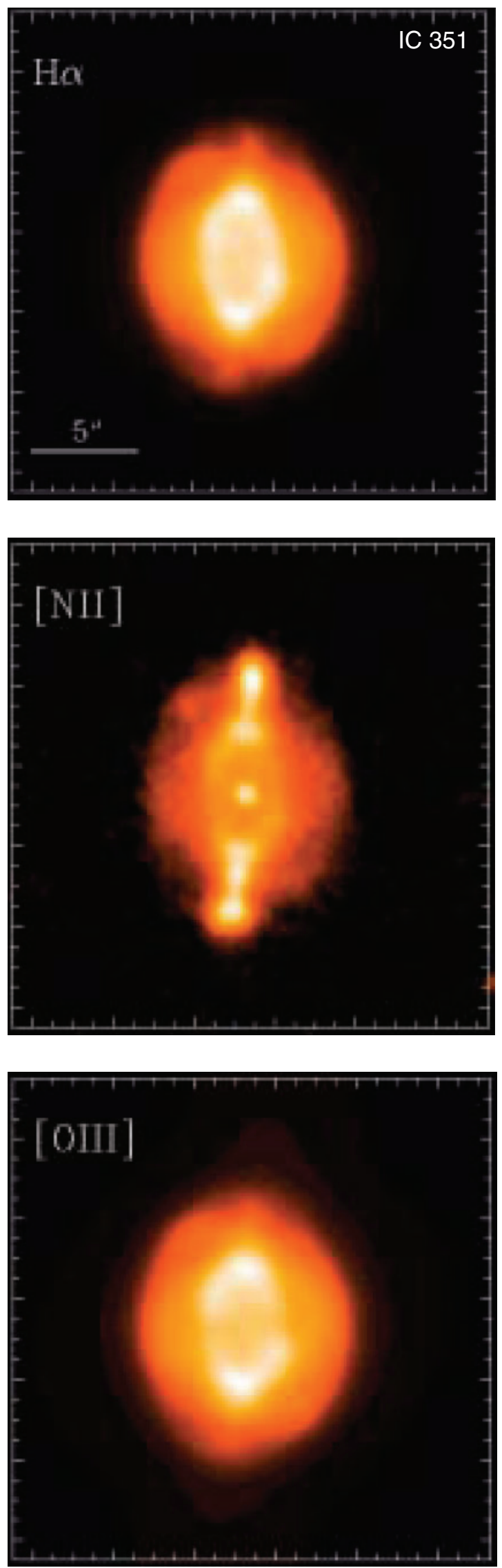

Figure $1 \mathrm{H} \alpha,[\mathrm{OIII}]$ and [NII] images of IC 351. North is up, east to the left. The images are displayed in a logarithmic scale. White represents high values of the intensity. 

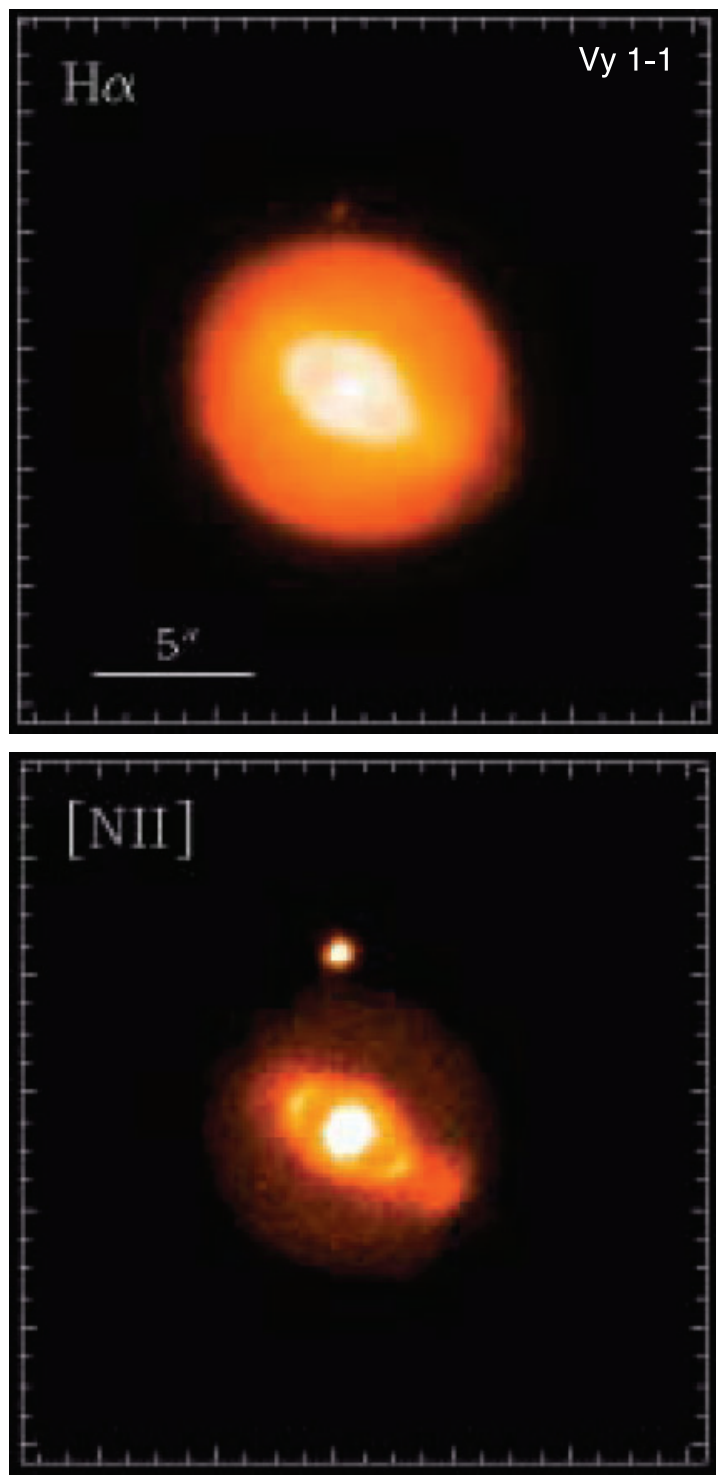

Figure $2 \mathrm{H} \alpha$ and [NII] images of Vy 1-1. North is up, east to the left. The images are displayed in a logarithmic scale. White represents high values of the intensity.

particularly bright in [NII]. Two polar knots are observed outside but connected to the elliptical inner shell in the [NII] image; they are not recognizable in the $\mathrm{H} \alpha$ image. The south-western knot is brighter than the north-eastern one.

IC 351 and Vy 1-1 closely resemble NGC 6826 and NGC 7009 (Balick et al. 1998). Although the radial velocities of the polar structures in IC 351 and Vy 1-1 have not been measured, it is probable that they represent FLIERs as in the case of NGC 6826 and NGC 7009. The morphological resemblances suggest similar formation processes in the four PNe.

\section{$3.2 N G C 6778$}

NGC 6778 is a PN that contains two bipolar jet systems oriented at different directions and moving at 100$200 \mathrm{~km} \mathrm{~s}^{-1}$ (Maestro et al. 2004). Figure 3 shows a colour
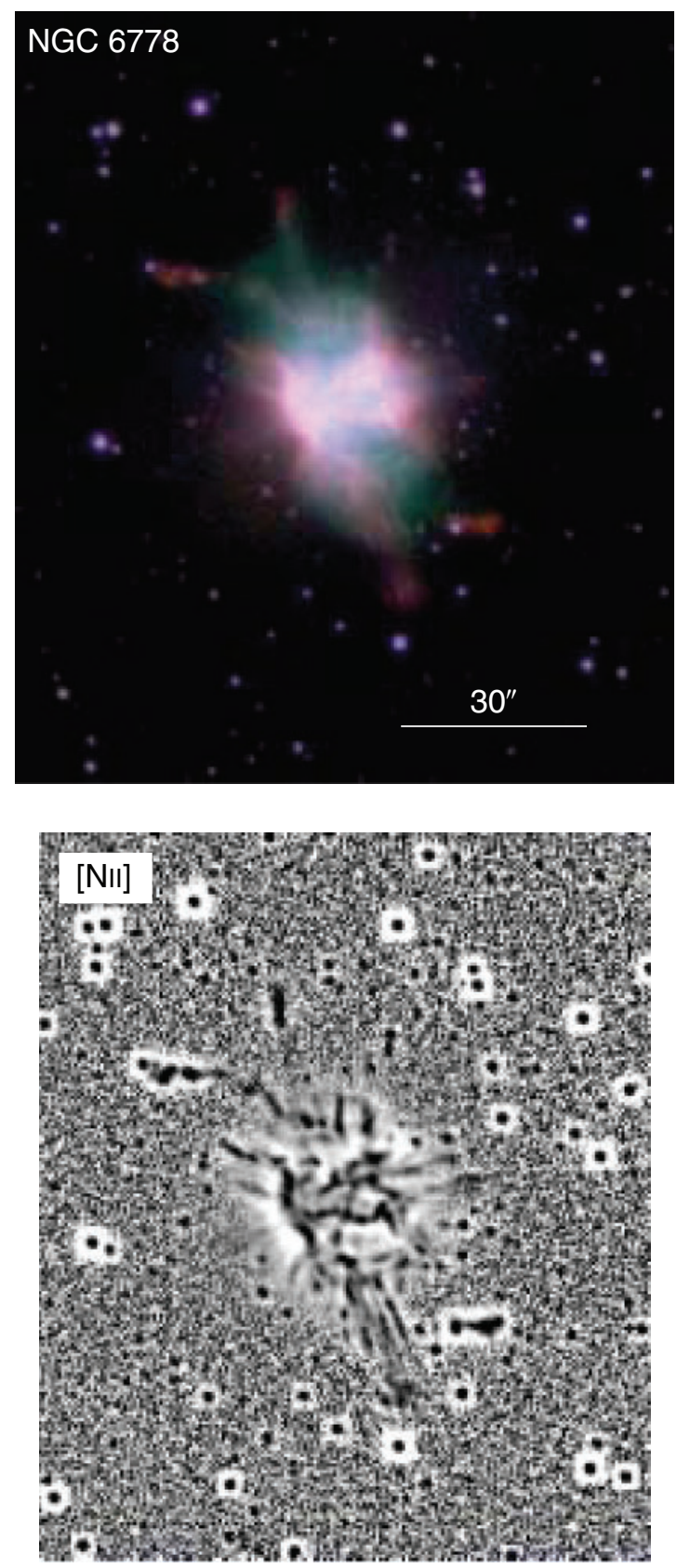

Figure 3 (Top) Colour composite picture of NGC6778 (green $=\mathrm{H} \alpha$, blue $=[\mathrm{OIII}]$ and red $=[\mathrm{NII}])$. The image is displayed in a logarithmic scale. (Bottom) Unsharp masking [NII] image. The image is displayed in a linear scale. North is up, east to the left in the images.

composite picture of NGC 6778 constructed with the $\mathrm{H} \alpha$, [OIII] and [NII] images. The high resolution of the new images allow us a detailed description of the nebulae. The appearance of NGC 6778 is that of a bipolar nebula with its major axis along $\mathrm{PA} \simeq 15^{\circ}$. The nebula does not display the characteristic waist of butterfly $\mathrm{PNe}$, but the region along its minor axis is defined by a fragmented structure that is particularly bright in [NII]. In the highexcitation [OIII] emission, the nebula appears elliptical rather than bipolar. The images reveal details of the bipolar jets. In particular, the jet oriented at $\mathrm{PA} \simeq 195^{\circ}$ consists 

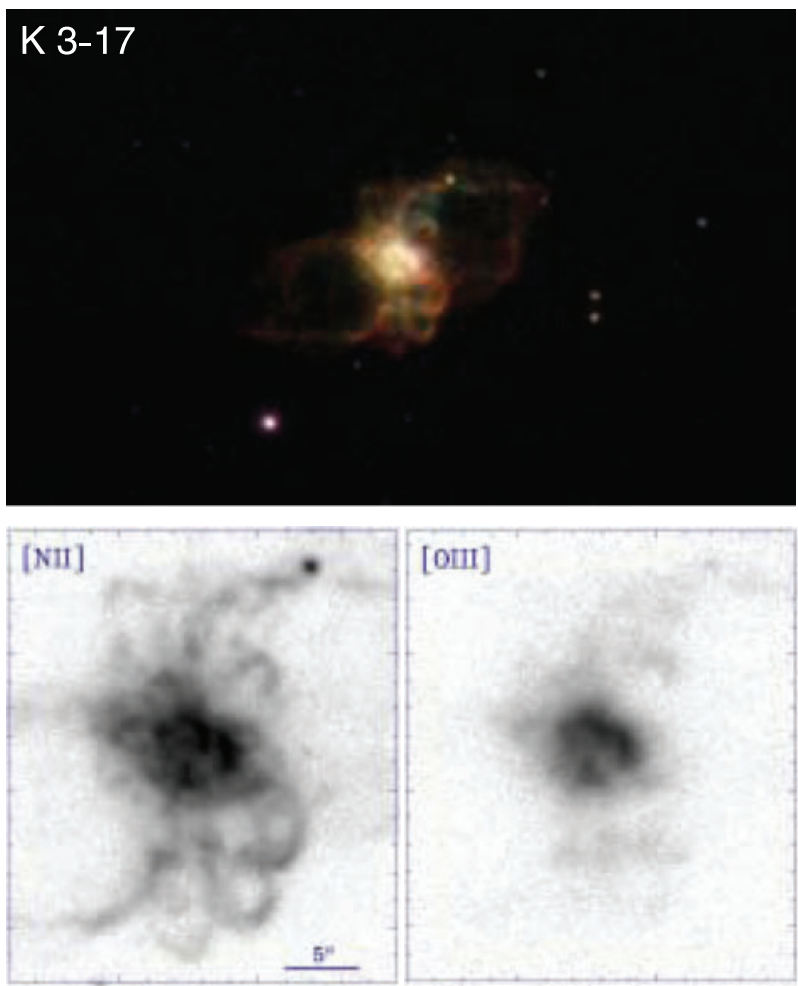

Figure 4 (Top) Colour composite picture of K 3-17 (green $=\mathrm{H} \alpha$, blue $=[\mathrm{OIII}]$ and red $=[\mathrm{NII}]) .($ Bottom $)$ Enlargement of the bright central region in the $[\mathrm{NII}]$ and [OIII] images. The images are displayed in a logarithmic scale. North is up, east to the left in the images. The spatial scale is indicated in the [NII] image.

of at least four filaments emanating from a bright knot. In addition, the nebula presents many low-excitation knots with a cometary appearance, embedded in high-excitation material. These knots are more clearly observed in the unsharp masking [NII] image also shown in Figure 3. This image further strengthens the complexity of the nebula. We found interesing similarities between NGC 6778 and Sh 2-71 (Bohigas 2001; Miranda et al. 2005) as both PNe exhibit extremely knotty and filamentary morphologies that suggests that the previously existing structures have been fragmented and probably swept up by the fast stellar wind.

\section{$3.3 \quad K 3-17$}

K 3-17 presents a bipolar morphology in the Manchado et al. (1996) catalog consisting of a bright compact core and two faint bipolar lobes in the $\mathrm{H} \alpha+[\mathrm{NII}]$ image. In the [OIII] image only the bright compact core was detected. Our new, higher resolution images reveal that K 3-17 is a complex PN with a wealth of structures. A colour composite picture is shown in Figure 4 along with [NII] and [OIII] images of the bright core. In the new images, the bipolar lobes present a spindle-like shape and are dominated by [NII] emission. The core is resolved into a series of bubbles oriented mainly perpendicular to the bipolar lobes, although small bubbles oriented along the bipolar axis are also observed. No ring-like or toroidal structure can be

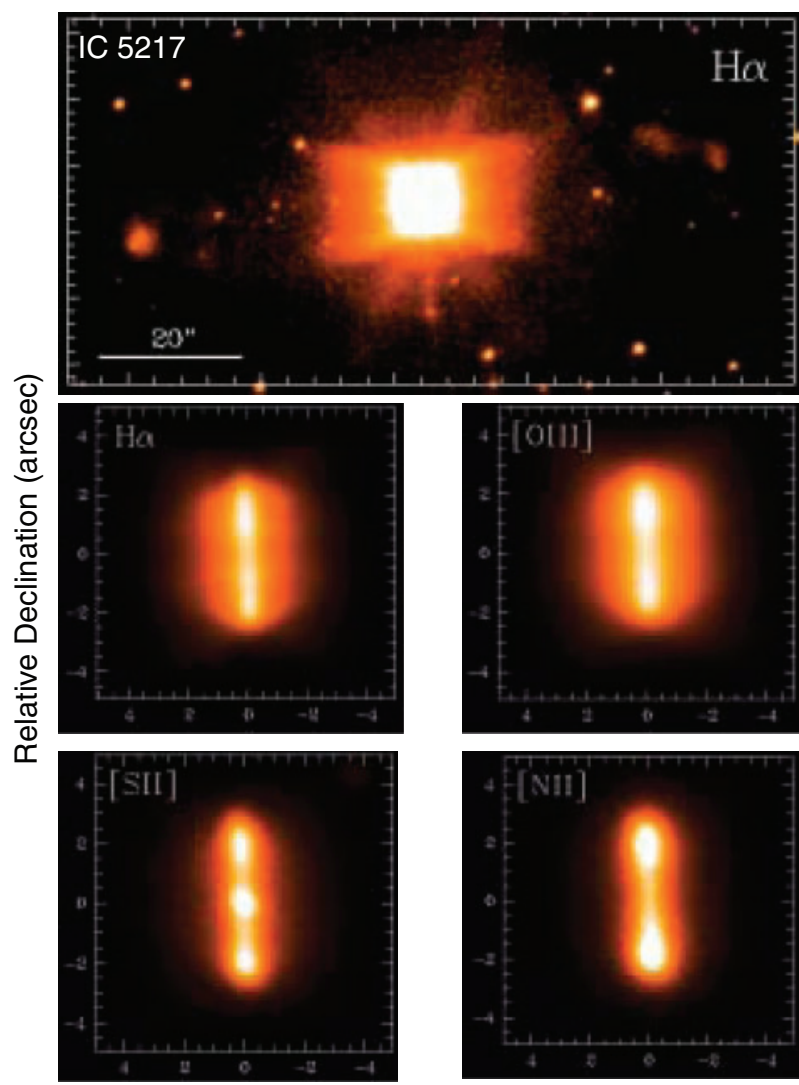

Relative Right Ascencion (arcsec)

Figure 5 (Top) $\mathrm{H} \alpha$ image of IC 5217 showing the whole bipolar structure and the point-symmetric features. North is up, east to the left. (Bottom) Images of the equatorial regions in several filters (upper left). The central star is observed in the [SII] filter. The images are displayed in a logarithmic scale.

identified in the center but bright knots without a particular orientation. Most of the structures are of low-excitation while high-excitation is only observed at the central knots and in the regions of the lobes near the center.

The spindle-like morphology of the lobes suggests the action of collimated outflows in their formation. A similar morphology is observed in $\mathrm{Hu} 2-1$ in which the distorsions of the lobes can be attributed due to the action of a bipolar collimated outflows (Miranda et al. 2001). As for the origin of the equatorial bubbles in K $3-17$, it could be possible that the stellar wind is protruding through or breaking an original equatorial structure. Alternatively, the bubbles may represent collimated outflows along different directions. In this case, the orientation of the collimation axis should have changed drastically by $\simeq 90^{\circ}$. Large differences in the orientation of multipolar lobes are also found in other PNe, being Sh 2-71 an extreme case (Miranda et al. 2005), while the equatorial bubbles in K 3-17 are similar to these observed in HST images of Hb 5 (see Montez et al. 2009).

\section{IC 5217 and KjPn 6}

IC 5217 is an edge-on bipolar PN with polar pointsymmetric features, a large axial ratio and a narrow waist that contains a very thin, bright equatorial ring (Miranda et al. 2006). 
Figure 5 presents a new $\mathrm{H} \alpha$ image and images of the ring in several emission lines. The large scale $\mathrm{H} \alpha$ image is similar to that presented by Miranda et al. (2006). In particular, the point-symmetric structures at large distances from the center are observed in this image as well as the bipolar lobes emanating from the nebular central regions that seem to be connected with the pointsymmetric features. The higher resolution of the new images and the observations in several emission line filters allow us to analyze the bright equatorial ring in great detail. Four local maxima along the ring are distinguished in the $\mathrm{H} \alpha$ image in agreement with the radio continuum morphology at $3.6 \mathrm{~cm}$ (see Miranda et al. 2006). In the rest of the emission lines, a clear ionization gradient is observed with the radius of the ring in [OIII] being smaller than in [NII] and [SII]. We also note that the central star is detected in the [SII] image at the center of the ring. It is worth noting that, if only a short exposure image would have been acquired, IC 5217 would be described as a highly collimated bipolar jet emanating from the central star. This is not the case as high-resolution, long-slit spectroscopy demonstrates that the bright structure is an edge-on ring (Miranda et al. 2006).

$\mathrm{KjPn} 6$ presents a peculiar, almost triangular shape in the $\mathrm{H} \alpha+[\mathrm{NII}]$ image and extremely faint emission in the [OIII] image (Manchado et al. 1996). The new images of $\mathrm{KjPn} 6$ are shown in Figure 6. The structure in $\mathrm{H} \alpha$ and [OII] resembles an elliptical PN with the major axis near the north-south direction, while in [NII] the nebula appears more squarish. Faint details are observed in the inner regions, which are particularly bright in [NII] and $\mathrm{H} \alpha$ but absent in [OIII]. The morphology of $\mathrm{KjPn} 6$ is very similar to this observed in the inner regions of IC 5217 (Figure 5), which have been identified with an edge-on ring. These similarities lead us to suggest that KjPn 6 may be a bipolar PN with bipolar much fainter than its equatorial regions. Therefore, IC 5217 and KjPn 6 are probably related to ring-like PNe that are characterized by the presence of a bright ring accompanied by faint bipolar lobes, although these are not always detected (e.g. Bond, Pollacco \& Webbink 2003). Interesting similarites are found with the edge-on ring-like PNe Me 1-1 (Pereira et al. 2008) and IC 2149 (Vázquez et al. 1999).

\section{Bipolar Planetary Nebulae}

Figure 7 shows colour composite pictures of six bipolar PNe. The new images are deeper and of higher quality than these published elsewhere. BV 1 is an edge-on bipolar with a bright ring and extremely faint bipolar lobes (Kaler et al. 1988). HaTr 10, M 4-17, and K 3-46 are likely similar to BV 1, the main difference being the orientation of the polar axis with respect to the observer and/or the evolutionary stage. The case of NGC 650 could be different as this PN is observed almost edge-on, as is the case of $\mathrm{BV} 1$, but the equatorial region is thick and there is not a large intensity contrast between this equatorial region and the bipolar lobes. In addition, NGC 650 shows bubbles and microstructures that suggest the presence of
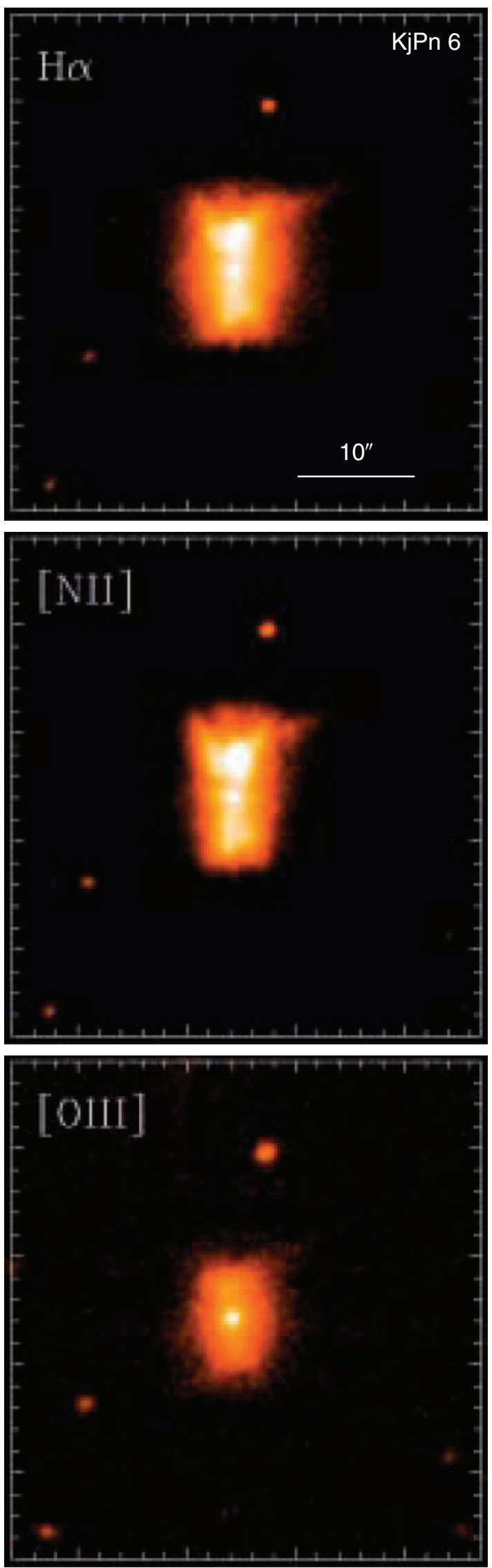

Figure $6 \mathrm{H} \alpha$, [NII] and [OIII] images of $\mathrm{KjPn} 6$. The images are displayed in a logarithmic scale. North is up, east to the left in each image. 

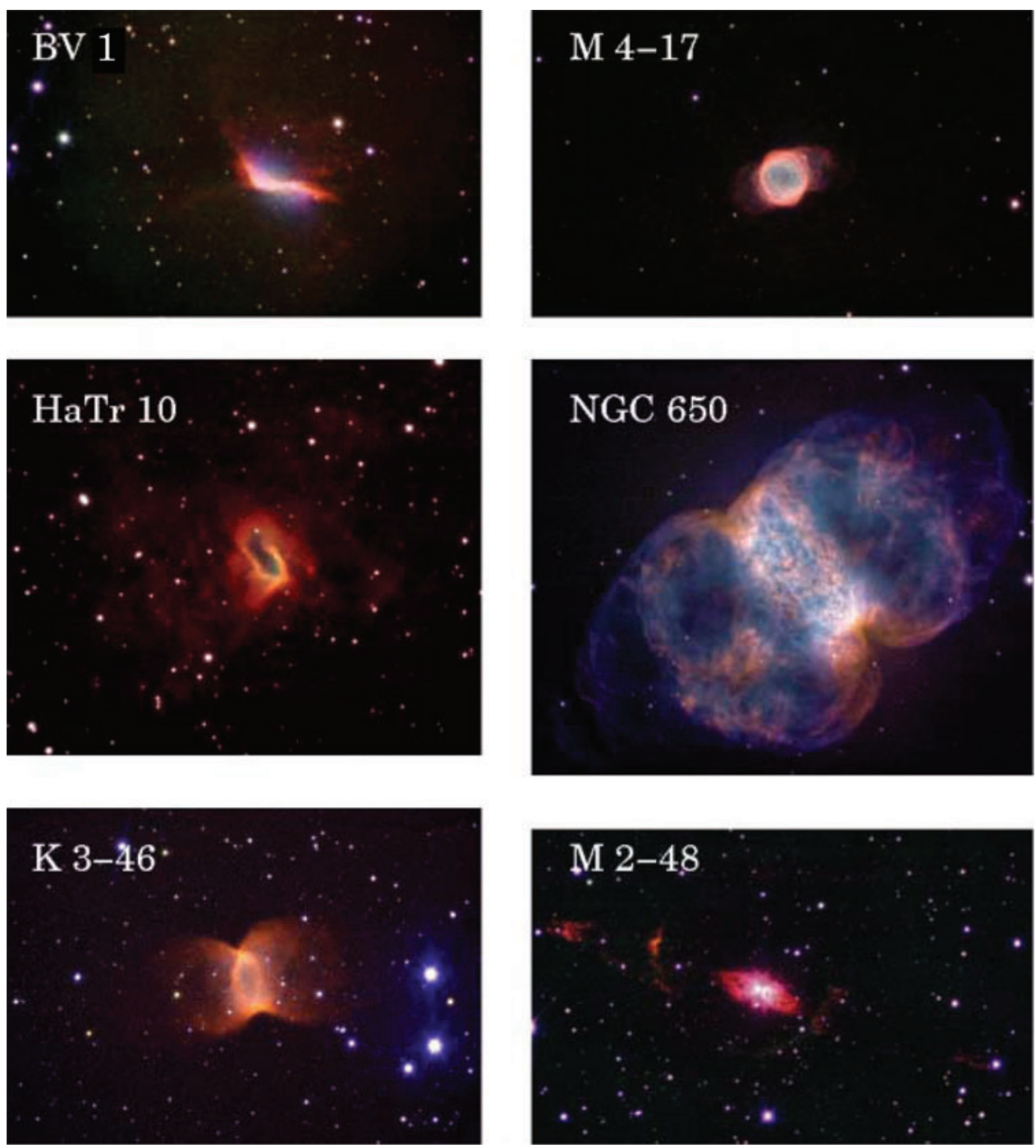

Figure 7 Colour composite pictures of BV 1 (field of view [fov] $\left.\simeq 285^{\prime \prime} \times 155^{\prime \prime}\right), \mathrm{M} 4-17$ (fov $\left.\simeq 255^{\prime \prime} \times 130^{\prime \prime}\right), \operatorname{HaTr} 10\left(\right.$ fov $\left.\simeq 170^{\prime \prime} \times 140^{\prime \prime}\right)$, NGC 650 (fov $\left.\simeq 130^{\prime \prime} \times 105^{\prime \prime}\right)$, K 3-46 (fov $\left.\simeq 215^{\prime \prime} \times 150^{\prime \prime}\right)$ and M 2-48 (fov $\left.\simeq 245^{\prime \prime} \times 155^{\prime \prime}\right)$. In all cases the colour code is green $=\mathrm{H} \alpha$, blue $=[\mathrm{OIII}]$ and red $=[\mathrm{NII}]$. The images are displayed in a logarithmic scale. North is up, east to the left in each image.

focused (collimated) outflows. M 2-48 is a bipolar PN with multiple structures and high velocity collimated outflows (Vázquez et al. 2000; López-Martín et al. 2002). Our high resolution image (Figure 7) shows in great detail the structures previously detected. A dark lane, rather than a bright equatorial torus or ring, separates the bipolar lobes. Elongated protrusions are observed along the minor axis of the nebula, although their orientation is different from this of the bipolar lobes and collimated outflows. A series of knots encircle the bipolar lobes tracing an apparent circular shell. This shell is brighter at four regions along the main symmetry axis and its center does not coincide with the nebular core. The distant outflows discovered by Vázquez et al. (2000) appear as knotty structures, particularly the north-eastern one. Its south-western counterpart is clearly detected in the new images, while only hints of it were found by Vázquez et al. (2000). M 2-48 presents noticeable differences when compared to the other bipolar PNe in Figure 7. On the other hand, the presence of distant outflows in M 2-48 resembles the situation observed in IC 5217 (see above).

\section{Final Remarks}

Images obtained at sub-arcsec resolution $\left(0.5^{\prime \prime}-0.9^{\prime \prime}\right)$ have revealed new morphological details in selected PNe. In IC 351 and Vy 1-1, low-excitation, jet-like features have been detected for the first time. The morphology of these two objects is very similar to this of well studied PNe like NGC 6826 and NGC 7009, strongly suggesting that the same processes have operated in the formation of these objects. In NGC 6778 and K 3-17 we found very complex structures. NGC 6778 consists of a large number of cometary knots and filaments with two systems of collimated outflows. K3-17 presents equatorial bubbles oriented at different directions. Evidence for collimated outflows in K 3-17 is provided by the spindle-like bipolar lobes. IC 5217 and KjPn 6 are bipolar PNe consisting in a bright edge-on equatorial ring and faint bipolar lobes. 
A comparison of the bipolar PNe in the sample suggests that the formation processes of NGC 6778, K 3-17 and M 2-48, implying the action of collimated outflows, may differ from this of NGC 650, K 3-46 and BV 1 which fit the expectations of the GISW model.

\section{Acknowledgments}

This work has been supported partially by AYA200501495 of the Spanish MEC (co-funded with FEDER funds) and AYA2008-01934 of the Spanish MICINN (cofunded with FEDER founds), and by grant FQM1747 of Consejería de Innovación, Ciencia y Empresa of Junta de Andalucía.

\section{References}

Balick, B., 1987, AJ, 94, 671

Balick, B. et al., 1998, AJ, 116, 360

Balick, B. \& Frank, A., 2002, ARA\&A, 40, 439

Bohigas, J., 2001, RMxAA, 37, 237

Bond, H. E., Pollacco, D. L. \& Webbink, R. F., 2003, AJ, 125, 260

Frank, A., Balick, B., Icke, V. \& Mellema, G., 1993, ApJ, 404, L25

Gieseking, F., Becker, I. \& Solf, J., 1985, ApJ, 295, L17

Goncalves, D. R., Corradi, R. L. M. \& Mampaso, A., 2001, ApJ, 547,302

Guerrero, M. A., Chu, Y.-H. \& Miranda, L. F., 2004, AJ, 128, 1694

Guerrero, M. A. et al., 2008, ApJ, 683, 272
Kaler, J. B., Chu, Y.-H. \& Jacoby, G. H., 1988, AJ, 96, 1407

Kwok, S., Purton, C. R. \& Fitzgerald, P. M., 1978, ApJ, 219, L125

López-Martín, L. et al., 2002, A\&A, 388, 652

Maestro, V., Guerrero, M. A. \& Miranda, L. F., 2004, ASPC, 313, 127 Manchado, A., Guerrero, M. A., Stanghellini, L. \& Serra-Ricart, M., 1996, The IAC Morphological Catalog of Northern Galactic Planetary Nebulae (La Laguna: Instituto de Astrofísica de Canarias)

Miranda, L. F., Ayala, S., Vázquez, R. \& Guillén, P. F., 2006, A\&A, 456, 591

Miranda, L. F., Ayala, S., Ulla, A., Olguín, L. \& Vázquez, R., 2005, in IAP Conference Proceedings, 804, Planetary Nebulae as Astronomical Tools, Eds. Szczerba, R., Stasińska, G. \& Górny, S. K. (New York: IAP), 97

Miranda, L. F., Guerrero, M. A. \& Torrelles, J. M., 1999, AJ, 117, 1421

Miranda, L. F. \& Solf, J., 1992, A\&A, 260, 397

Miranda, L. F., Torreles, J. M., Guerrero, M. A., Vázquez, R. \& Gómez, Y., 2001, MNRAS, 321, 487

Montez, R., Kastner, J. H., Balick, B. \& Frank, A., 2009, ApJ, 694, 1481

Pereira, C. B., Miranda, L. F., Smith, V. V. \& Cunha, K., 2008, A\&A, 477,535

Sahai, R. \& Trauger, J. T., 1998, AJ, 116, 1357

Schwarz, H. E., Corradi, R. L. M. \& Melnick, J., 1992, A\&AS, 96, 23

Stanghellini, L., Corradi, R. L. M. \& Schwarz, H. E., 1993, A\&A, 279,521

Vázquez, R. et al., 2000, A\&A, 357, 1031

Vázquez, R. et al., 2002, ApJ, 576, 860 